\title{
Chalcogen-nitrogen Bond: Insights into A Key Chemical Motif
}

\author{
Marco Bortoli, ${ }^{1}$ Andrea Madabeni, ${ }^{1}$ Pablo Andrei Nogara, ${ }^{2}$ Folorunsho B. Omage, ${ }^{2}$ Giovanni \\ Ribaudo, ${ }^{3}$ Davide Zeppilli, ${ }^{1}$ Joao Batista Teixeira Rocha, ${ }^{2 *}$ Laura Orian ${ }^{1 *}$ \\ 1 Dipartimento di Scienze Chimiche Università degli Studi di Padova Via Marzolo 135131 Padova, Italy; \\ marco.bortoli@unipd.it (M.B.); andrea.madabeni@studenti.unipd.it (A.M..); \\ davide.zeppilli@studenti.unipd.it(D.Z.) \\ 2 Departamento de Bioquímica e Biologia Molecular, Universidade Federal de Santa Maria, Santa Maria, \\ 97105-900, RS Brazil; pbnogara@gmail.com (P.A.N.); omagefolorunsho@gmail.com (F.B.O.) \\ 3 Dipartimento di Medicina Molecolare e Traslazionale, Università degli Studi di Brescia, Viale Europa 11, \\ 25123 Brescia, Italy; giovanni.ribaudo@unibs.it (G.R.) \\ * Correspondence: : jbtrocha@gmail.com (J.B.T.R), laura.orian@unipd.it (L.O.); \\ + Presented at the 1st International Electronic Conference on Catalysis Sciences, 10-30 November 2020; \\ Available online: https://eccs2020.sciforum.net/
}

Published: 10 November 2020

\begin{abstract}
Chalcogen-nitrogen chemistry deals with systems in which sulfur, selenium or tellurium is linked to a nitrogen nucleus. This chemical motif is a key component of different functional structures, ranging from inorganic materials and polymers to rationally designed catalysts, to bioinspired molecules and enzymes. The formation of a selenium-nitrogen bond, and its disruption, are rather common events in organic Se-catalyzed processes. In nature, along the mechanistic path of glutathione peroxidase, evidence of the formation of a Se-N bond in highly oxidizing conditions has been reported and interpreted as a strategy to protect the selenoenzyme from overoxidation. Selenium is bonded to nitrogen also in the well-known ebselen, a selenenylamide with antioxidant, antimicrobic and cytoprotective activity and its formation/disruption has a crucial role for its pharmacological action. Focusing on examples taken from selenium organic chemistry and biochemistry, the selenium-nitrogen bond is described, and its strength and reactivity are quantified using accurate computational methods applied to model molecular systems. Significant trends show up when comparing to sulfur/tellurium-nitrogen bonds, reaffirming also in this context the peculiar and valuable role of selenium in chemistry and life
\end{abstract}

Keywords: selenium; nitrogen; ebselen; selenazoles; DFT calculations; molecular orbitals

\section{Introduction}

The chalcogen-nitrogen bond $(X-N, X=S, S e, T e)$ is an important motif in chemistry present in many different structures, ranging from inorganic and organic materials, catalysts, protein mimics, and potential drugs [1-4]. In the lab, the formation of a $\mathrm{X}-\mathrm{N}$ bond is typically obtained from benzanilide, using ortholithiation, chalcogen insertion, and oxidative cyclization [5-8], and by intramolecular cyclocondensation of chalcogenic acids [9-11].

The Se-N bond is present in the isoselenazole ring of ebselen (2-phenyl-1,2-benzisoselenazol$3(2 \mathrm{H})$-one), which is described as the oldest and most popular glutathione peroxidase (GPx) mimic. So far, the mechanistic details of its catalytic activity have not been fully elucidated [12-16], although a nice computational investigation has provided an exhaustive picture of the possible paths [17], disclosing that the chemistry of the Se-N bond plays a crucial role in triggering the antioxidant effects $[3,18]$. 
Importantly, it was recently demonstrated that ebselen is a potent inhibitor of the SARS-CoV-2 main protease (Mpro), suggesting its potential therapeutic use for COVID-19. The reaction between the thiol moiety from the cysteine residue of Mpro active site and the isoselenazole ring can break the Se-N bond, leading to the formation of a Se-S bond and subsequent enzyme inhibition $[19,20]$.

An early overview on the structure and on the electronic as well as steric features of $\mathrm{Se}-\mathrm{N}$ and $\mathrm{Te}-\mathrm{N}$ bonds was reported by Bjorgvinsson and Roesky three decades ago [21]. The structures of several X-N bond-containing heterocycles were also reported. Mahmudov and colleagues provided an updated perspective on the relevance of covalent and non-covalent chalcogen bonding in several trending branches of modern solid state and solution chemistry. Organic and inorganic synthesis, catalysis, material sciences and molecular recognition are just some of the fields in which $\mathrm{X}-\mathrm{N}$ bond can direct and assist chemical reactivity [22].

In biochemistry and medicinal chemistry, besides ebselen and other GPx mimics, compounds based on heterocyclic scaffolds containing one or multiple Se-N bonds are being studied as antibiotic, antifungal, anticancer, anti-inflammatory, analgesic, and antimicrobial agents [1,3,14]. Particularly, selenazolinium salts appear as promising candidates in light of their good reactivity and selectivity towards thiol groups in peptides, proteins and enzymes [23].

In this work, the $\mathrm{X}-\mathrm{N}$ bond is described and its strength and reactivity changes upon varying the chalcogen are quantified using accurate computational methods applied to model molecular systems, which enable to draw conclusions of general validity for the very different systems above described.

\section{Methods}

All calculations were performed with the Amsterdam Density Functional (ADF) program [24,25]. Geometry optimizations were performed with the GGA BLYP functional [26-29] in conjunction with a triple- $\zeta$ slater basis set with a double set of polarization functions (TZ2P). Core electron were kept frozen: up to 1 s for $C$ and $N$, up to $2 p$ for $S$, up to $3 p$ for $S e$, and up to $4 p$ for Te and the zeroth-order regular approximation (ZORA) was used to take relativistic effects into account [30-33]. Grimme's empirical dispersion correction with Becke-Johnson damping was added to correct the underestimation of non-bonded forces by this functional [34]. This level of theory will be referred as ZORA-BLYP-D3(BJ)/TZ2P. For the bonding analysis, based on the activation strain model [35,36], a more accurate level of theory was employed, i.e. using a full electron quadruple- $\zeta$ slater basis set (QZ4Pae). Moreover, the bonding analysis was carried out with the meta-hybrid M06-2X functional $[37,38]$ due to its excellent performance with radical systems [39]. Single points calculation done at this level of theory are denoted as ZORA-M06-2X/QZ4Pze//ZORA-BLYP-D3(BJ)/TZ2P. The X-N bond strength was calculated as the interaction energy between the two molecular fragments obtained after homolytic cleavage.

\section{Results}

The model chosen for the analysis of the chalcogen-nitrogen bonds have general formula RX$\mathrm{NR}_{2}{ }_{2}$ in which $\mathrm{X}$ can be $\mathrm{S}$, Se or Te and $\mathrm{R}$ and $\mathrm{R}^{\prime}$ vary among $\mathrm{H}, \mathrm{CH}_{3}$ and $\mathrm{CF}_{3}$. A selection of optimized molecular structures is reported in Figure 1.

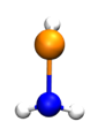

1

$\mathrm{R}=\mathrm{R}^{\prime}=\mathrm{H}$

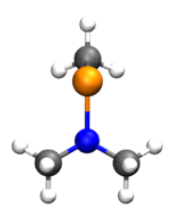

2

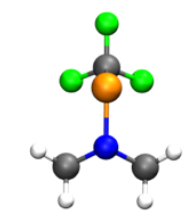

3

$\mathrm{R}=\mathrm{R}^{\prime}=\mathrm{CH}_{3}$

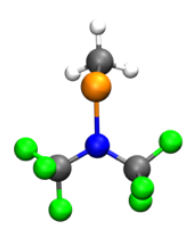

4

$\mathrm{R}=\mathrm{CH}_{3} \mathrm{R}^{\prime}=\mathrm{CF}_{3}$

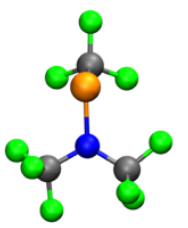

5

$\mathrm{R}=\mathrm{R}^{\prime}=\mathrm{CF}_{3}$

Figure 1. Optimized geometries of selected model molecules; $X=S e$. 
Analysis of the optimized geometries showed how the $\mathrm{X}-\mathrm{N}$ bond length vary upon changing the chalcogen or the substituents $\mathrm{R}$ and $\mathrm{R}^{\prime}$. Particularly, descending along the group we assist at a lengthening of the bond with all substituents, caused by the increase of the van der Waals radius of the increasingly bigger chalcogen. The variation upon modification of the substituents in series with the same chalcogen is smaller and does not follow the same trend for all the chalcogens. However, the structures showing the longest and shortest bond length are the same for all the chalcogens with the combination having $\mathrm{CF}_{3}$ on the chalcogen and $\mathrm{CH}_{3}$ on the nitrogen displaying the shortest bond length, whereas the specular combination, i.e. $\mathrm{R}_{2} \mathrm{R}^{\prime}=\mathrm{CH}_{3}, \mathrm{CF}_{3}$ leads to the longest bond length (e.g in the case of selenium the values are 1.86 and $1.92 \AA$ ).

To get a more exhaustive picture on the variation of the $\mathrm{X}-\mathrm{N}$ bond strength upon changing the chalcogen or substituent, an accurate analysis on the interaction energy between the two molecular fragments obtained after homolytic cleavage of the $\mathrm{X}-\mathrm{N}$ bond was carried out at two different levels of theory. The reaction investigated was:

$$
\mathrm{RX}-\mathrm{NR}_{2}{ }_{2} \rightarrow \mathrm{RX} \cdot+\cdot \mathrm{NR}_{2} \quad\left(\mathrm{X}=\mathrm{S} \text {, Se or Te; } \mathrm{R}, \mathrm{R}^{\prime}=\mathrm{H}, \mathrm{CH}_{3} \text { or } \mathrm{CF}_{3}\right)
$$

Results, reported in Table 2, show how the interaction diminishes as the chalcogen atom increases in size. For example, with $\mathrm{R}, \mathrm{R}^{\prime}=\mathrm{CH}_{3}$, it goes from $-65.5 \mathrm{kcal} \mathrm{mol}^{-1}$ in presence of sulfur to 42.6 in presence of tellurium. The same trend is found for all the substituents combination, and also when energies are computed with the accurate M06-2X functional combined with the QZ4Pae basis set (Table 1).

Table 1. $\triangle$ Eint for the model molecules. Structure definitions are reported in Figure 1. Level of theory: ZORA-M06-2X/QZ4Pze// ZORA-BLYP-D3(BJ)/TZ2P.

\begin{tabular}{cccccc}
\hline RX-NR' & & \multicolumn{4}{c}{$\Delta$ Eint } \\
\hline Molecule & $\mathbf{R}$ & $\mathbf{R}^{\prime}$ & $\mathbf{S}$ & Se & Tel \\
\hline $\mathbf{1}$ & $\mathrm{H}$ & $\mathrm{H}$ & -73.0 & -59.8 & -53.7 \\
$\mathbf{2}$ & $\mathrm{CH}_{3}$ & $\mathrm{CH}_{3}$ & -65.5 & -50.2 & -42.6 \\
$\mathbf{3}$ & $\mathrm{CF}_{3}$ & $\mathrm{CH}_{3}$ & -71.9 & -56.2 & -45.1 \\
$\mathbf{4}$ & $\mathrm{CH}_{3}$ & $\mathrm{CF}_{3}$ & -77.6 & -66.9 & -63.9 \\
$\mathbf{5}$ & $\mathrm{CF}_{3}$ & $\mathrm{CF}_{3}$ & -75.4 & -63.5 & -59.5 \\
\hline
\end{tabular}

If we focus on the effect of the substituent effect, a clear trend is found that is common for all the chalcogens. The interaction energy becomes progressively less negative in the sequence $\mathbf{4}<\mathbf{5}<\mathbf{1}<\mathbf{3}$ $<2$ (Figure 2).

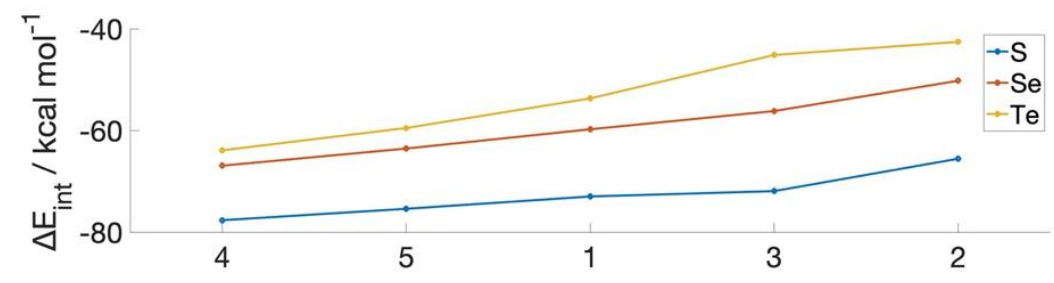

Figure 2. $\Delta$ Eint upon substituent variation. Level of theory ZORA-M06-2X/QZ4Pae//ZORA-BLYP$\mathrm{D} 3(\mathrm{BJ}) / \mathrm{TZ} 2 \mathrm{P}$.

\section{Discussion}

The orbital analysis of the radical fragments obtained after homolytic cleavage of the $\mathrm{X}-\mathrm{N}$ bond shows that upon variation of the chalcogen atom along the group, we assist to a decrease of the interaction energy, which becomes less negative, due to the fact that the fragment containing the chalcogen has its SOMO orbital that gets progressively more diffused. This makes the fragment "softer". On the other hand, the amino fragment contains a "hard" center, i.e. the $\mathrm{N}$ atom, and this 
worsens the interaction with the heavier chalcogens. The same behavior is found with all the different substituents, upon variation of the chalcogen.

The variation of the substituent on the interaction energy can be rationalized analyzing the effects that the different moieties have on the orbitals responsible for the $\mathrm{X}-\mathrm{N}$ bond. This translates to differently stabilized partially filled orbitals in the chalcogen and nitrogen molecular fragments depending on the substituent they contain. Electron donating substituents (e.g. $\mathrm{CH}_{3}$ ) give rise to fragments with higher energy frontier orbitals, whereas electron withdrawing ones (e.g. $\left.\mathrm{CF}_{3}\right)$ form fragments with lower frontier energy orbitals. Moreover, energetical separation of the two SOMOs is related to $\Delta \mathrm{E}$ int and the calculations nicely show that if the gap between the two SOMOs increases the interaction energy becomes more stabilizing, due to the formation of a more stabilized filled orbital.

\section{Conclusions}

In this work, we have analyzed with modern quantum mechanical techniques the nitrogenchalcogen bond. The results show that sulfur has a better interaction with nitrogen than selenium or tellurium due to the higher "hardness" of sulfur with respect to the other chalcogens. Besides, a correlation between the electron donating and electron withdrawing character of the substituents and the orbital energy of the molecular fragments can explain the different interaction energy calculated for the different species. These results can open new perspectives on the fine tuning of the chalcogennitrogen bond amenable of application in many biological and pharmaceutical studies targeted towards the development of novel bio-active compounds and antioxidant drugs.

Author Contributions: Conceptualization, supervision, validation and project administration, L.O. and J.B.T.R.; methodology, L.O, M.B.; formal analysis, investigation and data curation, A.M., D.Z., F.B.O; writing-original draft preparation, M.B., L.O., G.R., P.A.N.; writing - review and editing, M.B., G.R., L.O, J.B.T.R.

Funding: This research was funded by the Università degli Studi di Padova, thanks to the P-DiSC (BIRD2018UNIPD) project MAD ${ }^{3 S}$ (Modeling Antioxidant Drugs: Design and Development of computer-aided molecular Systems); P.I. L.O. P.A.N. and J.B.T.R was funded by CAPES (Edital 09-88887.505377/2020-00); F.B.O. was funded by CAPES (Edital 88887.354370/2019-00).

Conflicts of Interest: The authors declare no conflict of interest.

\section{References}

1. Joshi, P.G.; More, M.S.; Jadhav, A.A.; Khanna, P.K. Materials and biological applications of 1,2,3selenadiazoles: a review. Mater. Today Chem. 2020, 16, 100255.

2. Alberto, E.E.; Nascimento, V. Do; Braga, A.L. Catalytic application of selenium and tellurium compounds as glutathione peroxidase enzyme mimetics. J. Braz. Chem. Soc. 2010, 21, 2032-2041.

3. Barbosa, N. V.; Nogueira, C.W.; Nogara, P.A.; De Bem, A.F.; Aschner, M.; Rocha, J.B.T. Organoselenium compounds as mimics of selenoproteins and thiol modifier agents. Metallomics 2017, 9, 1703-1734.

4. Chivers, T.; Laitinen, R.S. Neutral binary chalcogen-nitrogen and ternary S,N,P molecules: New structures, bonding insights and potential applications. Dalt. Trans. 2020, 49, 6532-6547.

5. Engman, L.; Hallberg, A. Expedient Synthesis of Ebselen and Related Compounds. J. Org. Chem. 1989, 54, 2964-2966.

6. Sarma, B.K.; Manna, D.; Minoura, M.; Mugesh, G. Synthesis, structure, spirocyclization mechanism, and glutathione peroxidase-like antioxidant activity of stable spirodiazaselenurane and spirodiazatellurane. J. Am. Chem. Soc. 2010, 132, 5364-5374.

7. Zade, S.S.; Panda, S.; Tripathi, S.K.; Singh, H.B.; Wolmershäuser, G. Convenient synthesis, characterization and GPx-like catalytic activity of novel ebselen derivatives. European J. Org. Chem. 2004, 3857-3864.

8. Kersting, B.; DeLion, M. Synthesis of benzisochalcogenol and -azole derivatives via ortho metalation of isophthalamides. Zeitschrift fur Naturforsch. B 1999, 54, 1042-1047. 
9. Bhowmick, D.; Mugesh, G. Introduction of a catalytic triad increases the glutathione peroxidase-like activity of diaryl diselenides. Org. Biomol. Chem. 2015, 13, 9072-9082.

10. Sarma, B.K.; Mugesh, G. Antioxidant Activity of the Anti-Inflammatory Compound Ebselen: A Reversible Cyclization Pathway via Selenenic and Seleninic Acid Intermediates. Chem. Eur.J. 2008, 14, 10603-10614.

11. Singh, V.P.; Singh, H.B.; Butcher, R.J. Synthesis and glutathione peroxidase-like activities of isoselenazolines. European J. Org. Chem. 2011, 3, 5485-5497.

12. Müller, A.; Cadenas, E.; Graf, P.; Sies, H. A novel biologically active seleno-organic compound-1. Glutathione peroxidase-like activity in vitro and antioxidant capacity of PZ 51 (Ebselen). Biochem. Pharmacol. 1984, 33, 3235-3239.

13. Sies, H. Ebselen, A selenoorganic compound as glutathione peroxidase mimic. Free Radic. Biol. Med. 1993, $14,313-323$.

14. Nogueira, C.W.; Rocha, J.B.T. Toxicology and pharmacology of selenium: Emphasis on synthetic organoselenium compounds. Arch. Toxicol. 2011, 85, 1313-1359.

15. Azad, G.K.; Tomar, R.S. Ebselen, a promising antioxidant drug: Mechanisms of action and targets of biological pathways. Mol. Biol. Rep. 2014, 41, 4865-4879.

16. Zhao, R.; Holmgren, A. A novel antioxidant mechanism of ebselen involving ebselen diselenide, a substrate of mammalian thioredoxin and thioredoxin reductase. J. Biol. Chem. 2002, 277, 39456-39462.

17. Antony, S.; Bayse, C.A. Modeling the mechanism of the glutathione peroxidase mimic ebselen. Inorg. Chem. 2011, 50, 12075-12084.

18. Dalla Tiezza, M.; Ribaudo, G.; Orian, L. Organodiselenides: Organic Catalysis and Drug Design Learning from Glutathione Peroxidase. Curr. Org. Chem. 2018, 23, 1381-1402.

19. Jin, Z.; Du, X.; Xu, Y.; Deng, Y.; Liu, M.; Zhao, Y.; Zhang, B.; Li, X.; Zhang, L.; Peng, C.; et al. Structure of Mpro from SARS-CoV-2 and discovery of its inhibitors. Nature 2020, 582, 289-293.

20. Sies, H.; Parnham, M.J. Potential therapeutic use of ebselen for COVID-19 and other respiratory viral infections. Free Radic. Biol. Med. 2020, 156, 107-112.

21. Bjorgvinsson, M.; Roesky, H.W. The structures of compounds containing selenium-nitrogen and tellurium-nitrogen bonds. Polyhedron 1991, 10, 2353-2370.

22. Mahmudov, K.T.; Kopylovich, M.N.; Guedes Da Silva, M.F.C.; Pombeiro, A.J.L. Chalcogen bonding in synthesis, catalysis and design of materials. Dalt. Trans. 2017, 46, 10121-10138.

23. Rendekova, J.; Vlasakova, D.; Arsenyan, P.; Vasiljeva, J.; Nasim, M.J.; Witek, K.; Dominguez-Alvarez, E.; Zeslawska, E.; Manikova, D.; Tejchman, W.; et al. The Selenium-Nitrogen Bond as Basis for Reactive Selenium Species with Pronounced Antimicrobial Activity. Curr. Org. Synth. 2017, 14, 1082-1090.

24. te Velde, G.; Bickelhaupt, F.M.; Baerends, E.J.; Fonseca Guerra, C.; van Gisbergen, S.J.A.; Snijders, J.G.; Ziegler, T. Chemistry with ADF. J. Comput. Chem. 2001, 22, 931-967.

25. ADF2019.103; SCM, Theoretical Chemistry, Vrije Universiteit: Amsterdam, The Netherlands, WWW.scm.com;

26. Becke, A.D. Density-functional exchange-energy approximation with correct asymptotic behavior. Phys. Rev. A 1988, 38, 3098-3100.

27. Lee, C.; Yang, W.; Parr, R.G. Development of the Colic-Salvetti correlation-energy formula into a functional of the electron density. Phys. Rev. B 1988, 37, 785-789.

28. Johnson, B.G.; Gill, P.M.W.; Pople, J.A. The performance of a family of density functional methods. J. Chem. Phys. 1993, 98, 5612-5626. 
29. Russo, T. V.; Martin, R.L.; Hay, P.J. Density functional calculations on first-row transition metals. J. Chem. Phys. 1994, 101, 7729-7737.

30. van Lenthe, E.; Baerends, E.J.; Snijders, J.G. Relativistic regular two-component Hamiltonians. J. Chem. Phys. 1993, 99, 4597-4610.

31. van Lenthe, E.; Baerends, E.J.; Snijders, J.G. Relativistic total energy using regular approximations. J. Chem. Phys. 1994, 101, 9783.

32. van Lenthe, E.; Snijders, J.G.; Baerends, E.J. The zero-order regular approximation for relativistic effects: The effect of spin-orbit coupling in closed shell molecules. J. Chem. Phys. 1996, 105, 6505-6516.

33. Van Lenthe, E. Geometry optimizations in the zero order regular approximation for relativistic effects. J. Chem. Phys. 1999, 110, 8943-8953.

34. Grimme, S.; Ehrlich, S.; Goerigk, L. Effect of the damping function in dispersion corrected density functional theory. J. Comput. Chem. 2011, 32, 1456-1465.

35. Bickelhaupt, F.M.; Baerends, E.J. Kohn-Sham Density Functional Theory: Predicting and Understanding Chemistry. In Reviews in Computational Chemistry; Lipkovitz, K.B., Boyd, D.B., Eds.; Wiley-VCH: New York, NY, USA, 2000, 15, pp. 1-86. ISBN 9780470125922.

36. Bickelhaupt, F.M.; Houk, K.N. Analyzing Reaction Rates with the Distortion/Interaction-Activation Strain Model. Angew. Chemie - Int. Ed. 2017, 56, 10070-10086.

37. Zhao, Y.; Truhlar, D.G. A new local density functional for main-group thermochemistry, transition metal bonding, thermochemical kinetics, and noncovalent interactions. J. Chem. Phys. 2006, 125, 194101.

38. Zhao, Y.; Truhlar, D.G. The M06 suite of density functionals for main group thermochemistry, thermochemical kinetics, noncovalent interactions, excited states, and transition elements: Two new functionals and systematic testing of four M06-class functionals and 12 other function. Theor. Chem. Acc. 2008, 120, 215-241.

39. Zhao, Y.; Truhlar, D.G. How Well Can New-Generation Density Functionals Describe the Energetics of Bond-Dissociation Reactions Producing Radicals? J. Phys. Chem. A 2008, 112, 1095-1099.

(C) 2020 by the authors; licensee MDPI, Basel, Switzerland. This article is an open access article distributed under the terms and conditions of the Creative Commons Attribution (CC-BY) license (http://creativecommons.org/licenses/by/4.0/). 